\title{
Exact solution for peristaltic flow of Jeffrey fluid model in a three dimensional rectangular duct having slip at the walls
}

\author{
Arshad Riaz ${ }^{\mathrm{a}, \mathrm{b}, *}$, S. Nadeem ${ }^{\mathrm{c}}$, R. Ellahi ${ }^{\mathrm{a}}$ and A. Zeeshan ${ }^{\mathrm{a}}$ \\ ${ }^{a}$ Department of Mathematics and Statistics, FBAS, IIU, Islamabad, Pakistan \\ ${ }^{\mathrm{b}}$ Department of Mathematics, University of Sargodha, Lahore, Pakistan \\ ${ }^{\mathrm{c}}$ Department of Mathematics, Quaid-i-Azam University, Islamabad, Pakistan
}

\begin{abstract}
In the present article, we tried to develop the exact solutions for the peristaltic flow of Jeffrey fluid model in a cross section of three dimensional rectangular channel having slip at the peristaltic boundaries. Equation of motion and boundary conditions are made dimensionless by introducing some suitable nondimensional parameters. The flow is considered under the approximations of low Reynolds number and long wavelength. Exact solution of the obtained linear boundary value problem is evaluated. However, the expression for pressure rise is calculated numerically with the help of numerical integration. All pertinent parameters are discussed through graphs of pressure rise, pressure gradient, velocity and stream functions. It is found that presence of slip at the walls reduces the flow velocity but increases the peristaltic pumping characteristics.
\end{abstract}

Keywords: Peristaltic flow, slip effects, jeffrey fluid, rectangular duct, exact solution

\section{Introduction}

The movement with in the living organism's body are described by muscular movement stimulated by neurons. There are two types of movements voluntary or involuntary. The involuntary movement can not be controlled by the will of organism himself. Normally in the human body, the peristalsis motion is the example of an involuntary wavelike contractions and relaxation of smooth muscles. It occurs in transport tubes like oesophagus, urine transport from kidney or some blood vessels. Peristaltic phenomenon has enormous applications in chemical industries, engineering and biomedical equipment. In food tubes, food bolus initiate the waves which activate excitatory neurons above

*Corresponding author: Arshad Riaz, Department of Mathematics and Statistics, FBAS, IIU, 44000 Islamabad, Pakistan. Tel.: +92 3006076036; E-mail: arshad.phdma03@iiu.edu.pk. the bolus and consequently initiate inhibitory neurons that relaxes smooth muscles below the bolus [1]. These waves may have long or short wavelengths. Inspired by this phenomenon many industries like roller and finger pumps and in blood filtration devices etc. uses this mechanism. Nowadays, it is a well accepted fact that most of the fluid used in industries and biomedical treatments are non-Newtonian in rheological properties. So many researchers have presented the flow phenomena of non-Newtonian fluids [2-4]. The idea about the peristaltic flows was presented by Shapiro [5] and later verified experimentally by Latham [6]. Application of peristaltic flow have attracted the interest of many scientists to analyze the flow of Newtonian and non-Newtonian fluids through flexible walls of channel, duct or tube [7-12].

Tretheway and Meinhart [13] have experimentally shown slip-flows. Choi et al. [14] have studied apparent slip flows in hydrophilic and hydrophobic 
microchannels. Lauga and Cossu [15] have examined the stability of slip channel flow. Srinivas et al. [16] presented the influence of slip conditions, wall properties and heat transfer on MHD peristaltic transport. Nadeem and Akram. [17] studies heat transfer in a peristaltic flow of MHD fluid with partial slip.

Recently, the peristaltic flows in rectangular channels have become a subject of interest due to their physical and complex mathematical structure. Only a limited number of works could be found in the literature [18-20] because of the inherent mathematical complexity. However, the peristaltic flow of nonNewtonian fluid in a rectangular channel with slip effects are not explored so far. Therefore the present work is concentrated to examine the slip effects in peristaltic flow of a Jeffrey fluid in a rectangular duct. Long wavelength and low Reynolds number are used, which simplify the governing three dimensional partial differential equations. The exact solutions of the reduced equations have been found with the help of eigen function expansion method. The physical behavior of involving parameters is discussed through graphs of pressure rise, pressure gradient, velocity and stream functions. Three dimensional analysis is also taken into account for velocity profile.

\section{Mathematical formulation}

Let us consider the slip effects at the peristaltic walls for peristaltic flow of an incompressible non-Newtonian (Jeffrey fluid) fluid in a rectangular channel. The width and height of the channel are $2 d$ and $2 a$, respectively. In the current geometry, the Cartesian coordinate system is taken in such a way that $x$-axis is taken along the axial direction, $y$-axis is taken along the lateral direction and $z$-axis is perpendicular to both of $x$ and $y$ (see Fig. 1). The flow is induced by the propagation of sinusoidal waves travelling in the direction parallel to the flow.

The peristaltic wave equations at the upper and lower walls of the rectangular duct are given by

$$
Z= \pm H(X, t)= \pm a \pm b \cos \left[\frac{2 \pi}{\lambda}(X-c t)\right],
$$

where $a$ and $b$ are the amplitudes of the waves, $\lambda$ is the wavelength, $c$ is the velocity of propagation, $t$ is the time and $X$ is the direction of wave propagation. We assume that the lateral velocity is zero as there is no change in lateral direction of the channel. The

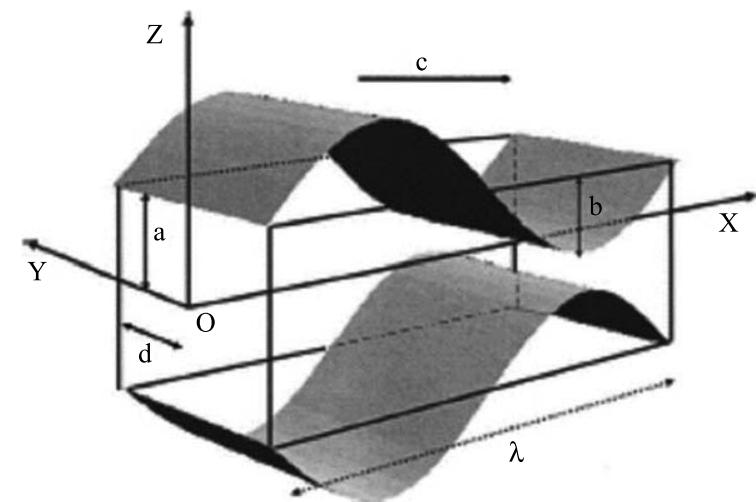

Fig. 1. Schematic diagram for peristaltic flow in a rectangular duct.

velocity profile for rectangular duct is described as $(U(X, Y, Z), 0, W(X, Y, Z))$. The governing equations for an incompressible fluid are defined as

$$
\begin{gathered}
\nabla \cdot \mathbf{V}=\mathbf{0}, \\
\rho \frac{D \mathbf{V}}{D t}=-\nabla P+\nabla \cdot \mathbf{S},
\end{gathered}
$$

where $D / D t$ is material time derivative, $\mathbf{S}$ represents the stress tensor for the Jeffrey model and is defined by [18]

$$
\mathbf{S}=\frac{\mu}{1+\lambda_{1}}\left(\dot{\gamma}+\lambda_{2} \ddot{\gamma}\right)
$$

In the above equation $\lambda_{1}$ is the ratio of relaxation to retardation times, $\lambda_{2}$ is the delay time, $\gamma$ is shear stress and double dots denote the differentiation with respect to time. Let us analyze the flow in a wave frame $(x, y, z)$ moving with a constant velocity $c$ away from the fixed frame $(X, Y, Z)$ by the transformation

$$
\begin{gathered}
x=X-c t, y=Y, z=Z, u=U-c, w=W, \\
p(x, z)=p(X, Z, t) .
\end{gathered}
$$

We define the following non-dimensional quantities

$$
\begin{aligned}
& \bar{x}=\frac{x}{\lambda}, \bar{y}=\frac{y}{d}, \bar{z}=\frac{z}{a}, \bar{t}=\frac{c}{\lambda} t, \bar{u}=\frac{u}{c}, \\
& \bar{w}=\frac{w}{c \delta}, \bar{h}=\frac{H}{a}, \beta=\frac{a}{d}, \delta=\frac{a}{\lambda}, \phi=\frac{b}{a}, \\
& \bar{p}=\frac{a^{2} p}{\mu c \lambda}, \overline{\mathbf{S}}=\frac{a}{\mu c} \mathbf{S}, \overline{\dot{\gamma}}=\frac{a}{c} \dot{\gamma}, \beta_{1}=\frac{L}{a}, \operatorname{Re}=\frac{\rho a c}{\mu} .
\end{aligned}
$$

Under the assumption of long wavelength $(\delta<<1)$ and low Reynolds number $(\operatorname{Re}<<1)$, the governing equations (2) and (3) in non-dimensional form for the 
considered flow problem are stated as (see Nadeem and Akram [18])

$$
\begin{aligned}
& \frac{\partial u}{\partial x}+\frac{\partial w}{\partial z}=0, \\
& \beta^{2} \frac{\partial^{2} u}{\partial y^{2}}+\frac{\partial^{2} u}{\partial z^{2}}=\left(1+\lambda_{1}\right) \frac{\partial p}{\partial x}, \\
& \frac{\partial p}{\partial y}=0=\frac{\partial p}{\partial z} .
\end{aligned}
$$

The corresponding slip boundary conditions [21-29] at the walls are described as retrieve the case for peristaltic flow of Jeffrey fluid in two dimensional channel, $\lambda_{1}=0$ gives the peristaltic flow of viscous fluid in three dimensional channel and $\beta_{1}=0$ leads to the no-slip conditions.

\section{Solution of the problem}

The exact solutions of the Eq. (10) along with boundary conditions defined in Eq. (9) have been evaluated by adopting the similar procedure as applied in [18] and is directly written as

$$
\begin{aligned}
u(x, y, z)= & \frac{-\frac{d p}{d x}\left(1-y^{2}+\lambda_{1}-y^{2} \lambda_{1}\right)-2 \beta^{2}}{2 \beta^{2}}+\sum_{n=1}^{\infty}\left(\cos \left(y \frac{\alpha_{n}}{\beta}\right)\right. \\
& \left(\operatorname { c o s h } ( z \alpha _ { n } ) \left(-\frac{8 \frac{d p}{d x}\left(1+\lambda_{1}\right)(2 \cos (n \pi))}{(-1+2 n)^{3} \pi^{3} \beta^{2}\left(\cosh \left(h \alpha_{n}\right)+\frac{\alpha_{n} \beta_{1} \sinh \left(h \alpha_{n}\right)}{1+\lambda_{1}}\right)}\right.\right. \\
& -\left(8\left(1+\lambda_{1}\right) \operatorname{sech}\left(h \alpha_{n}\right)\left(\frac{\alpha_{n} \beta_{1} \cosh \left(h \alpha_{n}\right)}{1+\lambda_{1}}+\sinh \left(h \alpha_{n}\right)\right)\right. \\
& \left.\left(2 \frac{d p}{d x} \alpha_{n} \beta_{1} \cos (n \pi) \sinh \left(h \alpha_{n}\right)\right)\right) /\left((-1+2 n)^{3} \pi^{3} \beta^{2}\right. \\
& \left(\alpha_{n} \beta_{1} \cosh \left(h \alpha_{n}\right)+\sinh \left(h \alpha_{n}\right)+\lambda_{1} \sinh \left(h \alpha_{n}\right)\right) \\
& \left.\left.\left(\cosh \left(h \alpha_{n}\right)+\frac{\alpha_{n} \beta_{1} \sinh \left(h \alpha_{n}\right)}{1+\lambda_{1}}\right)\right)\right)+\left(8\left(1+\lambda_{1}\right) \operatorname{Sech}\left(h \alpha_{n}\right)\right. \\
& \left.\left(2 \frac{d p}{d x} \alpha_{n} \beta_{1} \cos (n \pi) \sinh \left(h \alpha_{n}\right)\right) \sinh \left(z \alpha_{n}\right)\right) /\left((-1+2 n)^{3} \pi^{3} \beta^{2}\right. \\
& \left.\left.\left.\left(\alpha_{n} \beta_{1} \cosh \left(h \alpha_{n}\right)+\sinh \left(h \alpha_{n}\right)+\lambda_{1} \sinh \left(h \alpha_{n}\right)\right)\right)\right)\right),
\end{aligned}
$$

$$
\begin{aligned}
u & =-1 \text { at } y= \pm 1 \\
u & =-\frac{\beta_{1}}{1+\lambda_{1}} \frac{\partial u}{\partial z}-1 \text { at } z=h(x)=1+\phi \cos (2 \pi x) \\
u= & \frac{\beta_{1}}{1+\lambda_{1}} \frac{\partial u}{\partial z}-1 \text { at } z=-h(x) \\
& =-1-\phi \cos (2 \pi x) .
\end{aligned}
$$

From Eq. (8), it is obvious that $p \neq p(y, z)$. Therefore, Eq. (7) can be written in more precise form as

$$
\beta^{2} \frac{\partial^{2} u}{\partial y^{2}}+\frac{\partial^{2} u}{\partial z^{2}}=\left(1+\lambda_{1}\right) \frac{d p}{d x} .
$$

Special cases: There are some special cases which can be derived from the above discussion i.e., for $\beta=0$, we where $\alpha_{n}=(2 n-1) \frac{\pi}{2} \beta, n=1,2,3, \ldots$. The volumetric flow rate $q$ is calculated as

$$
q=\int_{0}^{h(x)} \int_{0}^{1} u(x, y, z) d y d z
$$

The average volume flow rate over one period $\left(T=\frac{\lambda}{c}\right)$ of the peristaltic wave is defined as

$$
Q=\int_{0}^{h(x)} \int_{0}^{1}(u(x, y, z)+1) d y d z=q+h(x) .
$$

The pressure gradient $d p / d x$ is obtained after solving Eqs. (12) and (13) and is found as 


$$
\begin{aligned}
\frac{d p}{d x}= & -\sum_{n=1}^{\infty}\left(Q /\left(\frac{h}{3 \beta^{2}}+\frac{h \lambda_{1}}{3 \beta^{2}}\right.\right. \\
& +\left(8 ( 1 + \lambda _ { 1 } ) 2 \operatorname { c o s } ( n \pi ) \operatorname { s i n } ( \frac { \alpha } { \beta } ) \left(\alpha_{n} \beta_{1}\right.\right. \\
& \left.\left.+\left(1+\lambda_{1}\right) \sinh \left(h \alpha_{n}\right)\right) \tanh \left(h \alpha_{n}\right)\right) / \\
& \left(( - 1 + 2 n ) ^ { 3 } \pi ^ { 3 } \alpha _ { n } ^ { 2 } \beta \left(\alpha_{n} \beta_{1}\right.\right. \\
& \left.\left.\left.\left.\cosh \left(h \alpha_{n}\right)+\left(1+\lambda_{1}\right) \sinh \left(h \alpha_{n}\right)\right)\right)\right)\right) .
\end{aligned}
$$

The pressure rise $\Delta p$ is evaluated by numerically integrating the pressure gradient $d p / d x$ over one wavelength, i.e.,

$$
\Delta p=\int_{0}^{1} \frac{d p}{d x} d x
$$

\section{Results and discussions}

We have obtained the exact analytical solutions for the peristaltic flow of Jeffrey fluid model in a rectangular channel having slip at the peristaltic walls. In this section, we discuss the effects of physical parameters on the rheological aspects of the considered flow. All the pertinent quantities are described through graphical treatment. Numerical variation of pressure rise and velocity profile is also described through Tables 1 and 2 Figs. 2-3 are drawn to show the effect of different parameters such as $\beta, \beta_{1}, \lambda_{1}$ and $\phi$ on pressure rise per unit wavelength $\Delta p$ against the mean flux $Q$. The fluid flow can be classify on the basis of coordinate system generated by taking $\Delta p$ along y-axis and $Q$ along x-axis in to three major regions: Quadrant (I) displays $Q>0$ and $\Delta p>0$ is called peristaltic pumping region, Quadrant (II) where $Q>0$ and $\Delta p<0$ is augmented flow region and Quadrant (IV) contains $Q<0$ and $\Delta p>0$ is known as backward pumping region. If $\Delta p=0$ it is called free pumping. Figure $2 a$ indicates free pumping at $Q=0$, an increase in $\beta$ rises the magnitude of pressure change in quadrant IV while reduces in II. Figure $2 b$ shows the behavior of $\Delta p$ with changing values of $\beta_{1}$. It is observed an increase in the values of $\beta_{1}$ gives opposite behavior as compared with $\beta$. Figure $3 a$ and $b$ is drawn to show for the variation of $\lambda_{1}$ and $\phi$ respectively. It is noted that with the increase in $\lambda_{1}$ pressure rise suppresses in augmented part while increased in backward pumping and for rising $\phi$ pressure change inversely.
Table 1

\begin{tabular}{lccc}
\multicolumn{4}{l}{ Variation of pressure rise $\Delta p$ for fixed $\lambda_{1}=0.1, \beta=0.5, \phi=0.1$} \\
\hline$Q$ & $\Delta p$ when $\beta_{1}=0$ & $\Delta p$ when $\beta_{1}=0.3$ & $\Delta p$ when $\beta_{1}=0.5$ \\
\hline-2.0 & 7.87572 & 6.13742 & 5.60602 \\
-11.6 & 6.30057 & 4.90993 & 4.48482 \\
-11.2 & 4.72543 & 3.68245 & 3.36361 \\
-0.8 & 3.15029 & 2.45497 & 2.24241 \\
-0.4 & 1.57514 & 1.22748 & 1.12120 \\
0.0 & 0.00000 & 0.00000 & 0.00000 \\
0.4 & -1.5751 & -1.2274 & -1.1212 \\
0.8 & -3.1502 & -2.4549 & -2.2424 \\
1.2 & -4.7254 & -3.6824 & -3.3636 \\
1.6 & -6.3005 & -4.9099 & -4.4848 \\
2.0 & -7.8757 & -6.1374 & -5.6060 \\
\hline
\end{tabular}

Table 2

Variation of velocity profile $u$ for fixed $\lambda_{1}=3, \beta=0.5, \phi=$ $0.2, Q=0.5, x=0, y=0.5$

\begin{tabular}{lccc}
\hline$z$ & $u$ when $\beta_{1}=0$ & $u$ when $\beta_{1}=0.3$ & $u$ when $\beta_{1}=0.5$ \\
\hline-1.2 & 0.48605 & 0.23108 & 0.09756 \\
-1.0 & 0.77306 & 0.52413 & 0.39374 \\
-0.8 & 0.99314 & 0.75071 & 0.62376 \\
-0.6 & 1.15593 & 0.92096 & 0.79799 \\
-0.4 & 1.26801 & 1.04190 & 0.92366 \\
-0.2 & 1.33366 & 1.11816 & 1.00559 \\
0.0 & 1.35529 & 1.15257 & 1.04679 \\
0.2 & 1.33366 & 1.14633 & 1.04872 \\
0.4 & 1.26801 & 1.09923 & 1.01143 \\
0.6 & 1.15593 & 1.00954 & 0.93353 \\
0.8 & 0.99314 & 0.87388 & 0.81212 \\
1.0 & 0.77306 & 0.68690 & 0.64245 \\
1.2 & 0.48605 & 0.44062 & 0.41739 \\
\hline
\end{tabular}

The variation in pressure gradient $d p / d x$ for different parameters $\beta, \beta_{1}, \lambda_{1}, \phi$ and $Q$ can be observed from Figs. 4-6. Figures $4 a$ and $b$ display effects of $\beta$ and $\beta_{1}$ respectively. Increase in $\beta$ decreases the magnitude of pressure gradient $d p / d x$ in the wavelength $x \in[0,1]$ whereas, increase in $\beta_{1}$ increases pressure gradient. In both the graphs the pressure at $x<0.2$ and $x>0.8$ attains a much smaller value indicating a low resistance to the flow and at about $x=0.5$ the maximum resistance to the flow is anticipated. Figure $5 a$ Shows a similar behavior for $\lambda_{1}$ as observed for $\beta_{1}$. Figure $5 b$ indicates that in the central region increase in amplitude ratio $\phi$ decreases the pressure but this behavior reverse itself in the region $x<0.2$ and $x>0.8$. Figure 6 shows decrease in pressure gradient with mean flow rate $Q$.

Next we examine the graphical aspects of the different parameters of interest on the velocity distribution through Figs. 7-11. The two and three dimensional analysis is submitted in these figures for all the observing parameters $\beta, \beta_{1}, \lambda_{1}, \phi$ and $Q$. The variation of $\beta$ is shown in Fig. 7. It is observed that velocity decreases 

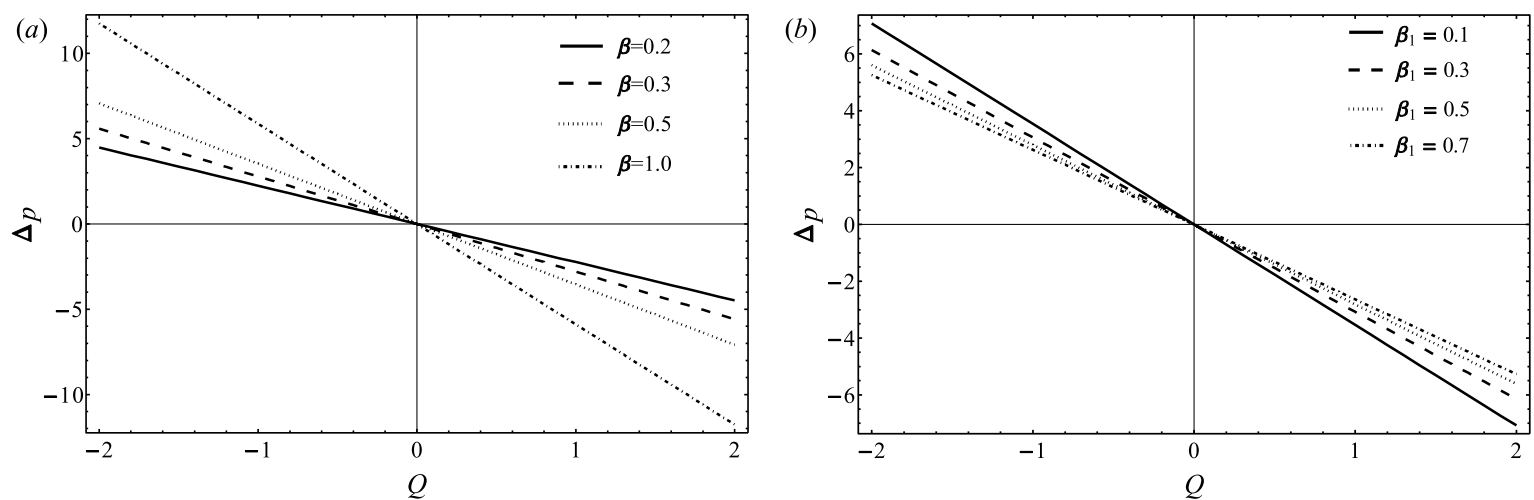

Fig. 2. (a) : Variation of $\Delta p$ for $\beta$ when other parameters are $\lambda_{1}=0.1, \beta_{1}=0.1, \phi=0.1$. (b) : Variation of $\Delta p$ for $\beta_{1}$ when other parameters are $\lambda_{1}=0.1, \beta=0.5, \phi=0.1$.
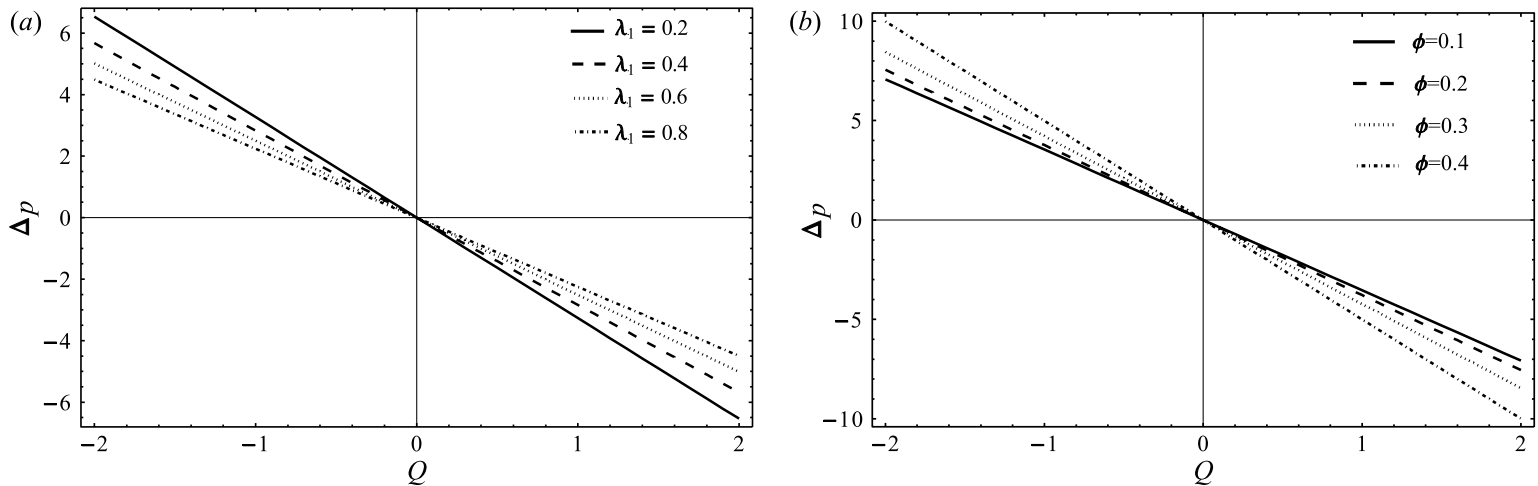

Fig. 3. (a) : Variation of $\Delta p$ for $\lambda_{1}$ when other parameters are $\beta=0.5, \beta_{1}=0.1, \phi=0.1$. $(b)$ : Variation of $\Delta p$ for $\phi$ when other parameters are $\lambda_{1}=0.1, \beta=0.5, \beta_{1}=0.1$.
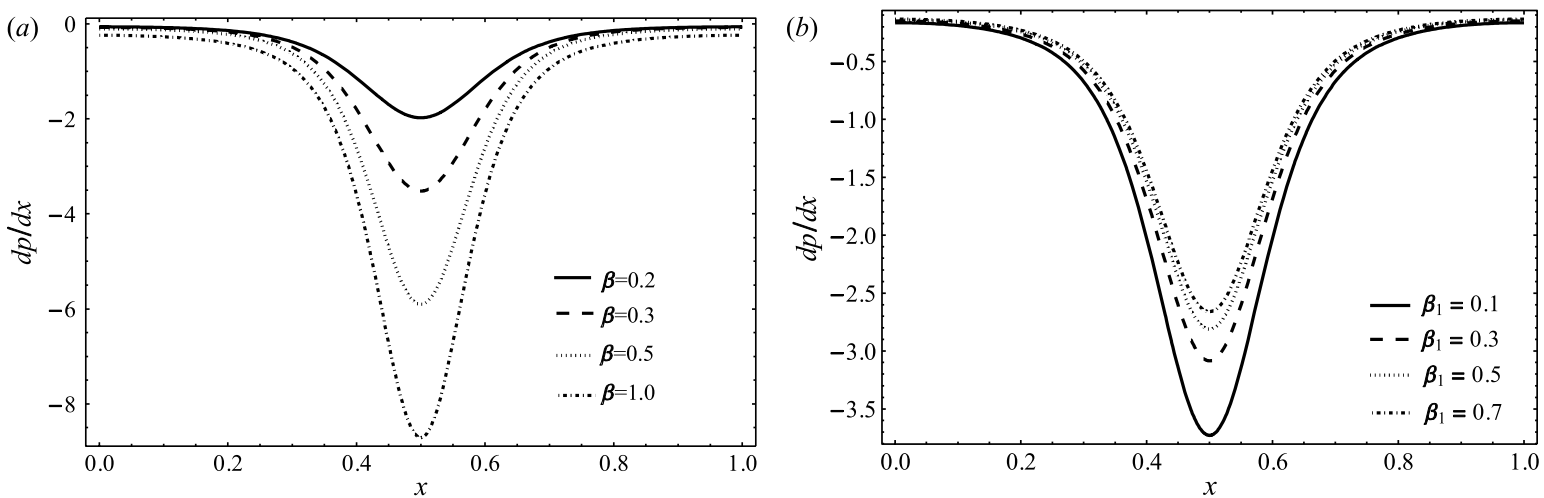

Fig. 4. (a) : Variation of $d p / d x$ for $\beta$ when other parameters are $\lambda_{1}=0.1, \beta_{1}=0.1, \phi=0.7, Q=0.1$. $(b):$ Variation of $d p / d x$ for $\beta_{1}$ when other parameters are $\lambda_{1}=0.1, \beta=0.7, \phi=0.6, Q=0.1$. 

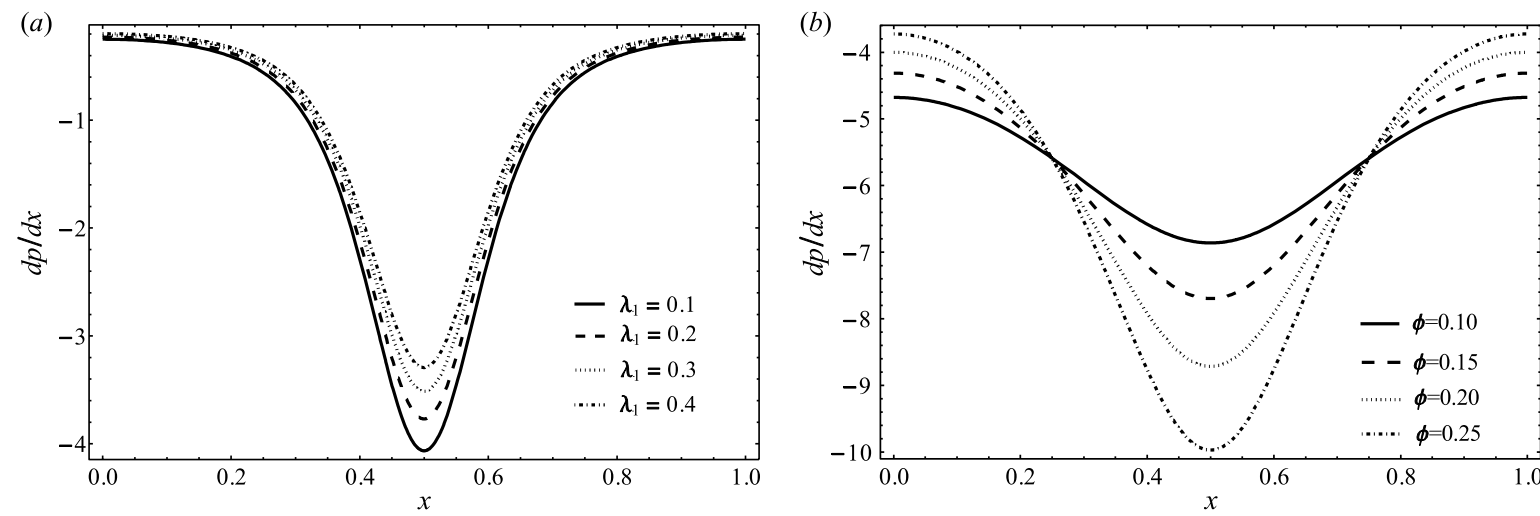

Fig. 5. (a) : Variation of $d p / d x$ for $\lambda_{1}$ when other parameters are $\beta=0.7, \beta_{1}=0.1, \phi=0.6, Q=0.1$. (b) : Variation of $d p / d x$ for $\phi$ when other parameters are $\lambda_{1}=0.1, \beta=0.7, \beta_{1}=0.1, Q=0.1$.

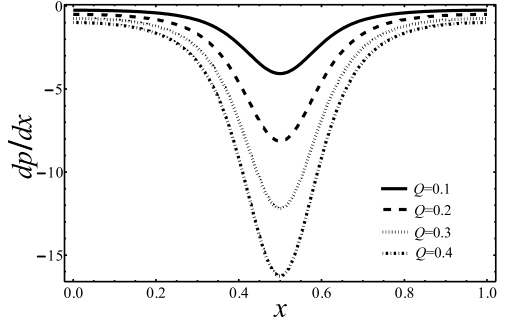

Fig. 6. Variation of $d p / d x$ for $Q$ when other parameters are $\beta=$ $0.7, \beta_{1}=0.1, \phi=0.6, \lambda_{1}=0.1$.

with the increasing effects of $\beta$. Figure 8 shows the variation of velocity for different fixed values of $\beta_{1}$. It is noted that in the part $z \in(-1,0.5)$, velocity decreases with the increase of $\beta_{1}$ while the situation is opposite in the rest of the region. The maximum velocity is at the centre of the duct but for smaller values of $\beta_{1}$ velocity decreases rapidly. The theoretical effects of $\lambda_{1}$ are dis- played in Figs. $9(a, b)$. An increase in velocity profile is observed when the values of $\lambda_{1}$ increases. Figures 10 and 11 show decrease in velocity when $\phi$ increases and increase when $Q$ increases respectively.

The stream lines are drawn in Figs. 12-15 to see the trapping bolus behavior with the variation of emerging parameters $\beta, \lambda_{1}, \phi$, and $Q$. From Fig. 12 , it is seen that numbers of the trapped bolus are reduced with increasing the values of the parameter $\beta$ where as the size increases. Figure 13 is for stream lines versus wavelength constructed for $\lambda_{1}$. It is measured here that number of bolus is reduced with the increase of $\lambda_{1}$. The stream lines for different values of the parameter $\phi$ are shown in Fig. 14. It is evident that the size of the trapped bolus increases with increasing $\phi$. It is measured from Fig. 15 that the size of trapped bolus decreases with mean flux $Q$, however the boluses are increased in number.
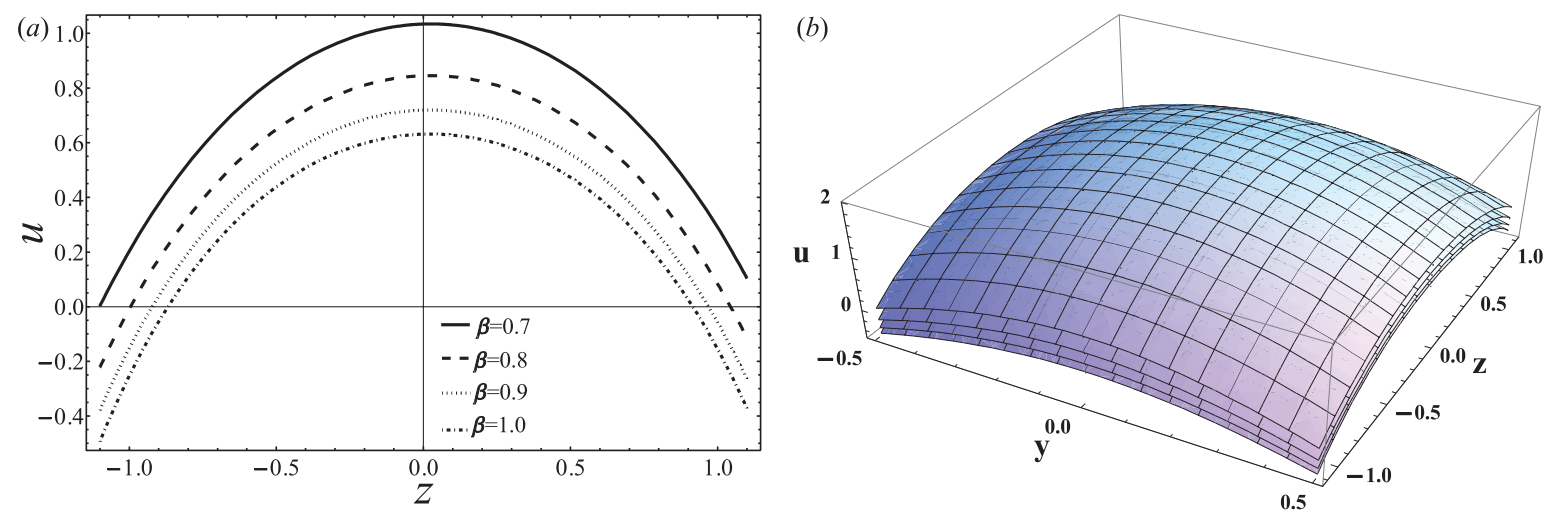

Fig. 7. Velocity profile for different values of $\beta$ for fixed $\phi=0.1, x=0, y=0.5, \beta_{1}=0.1, \lambda_{1}=3, Q=0.5$, $(a)$ for $2-$ dimensional, $(b)$ For 3-dimensional. 

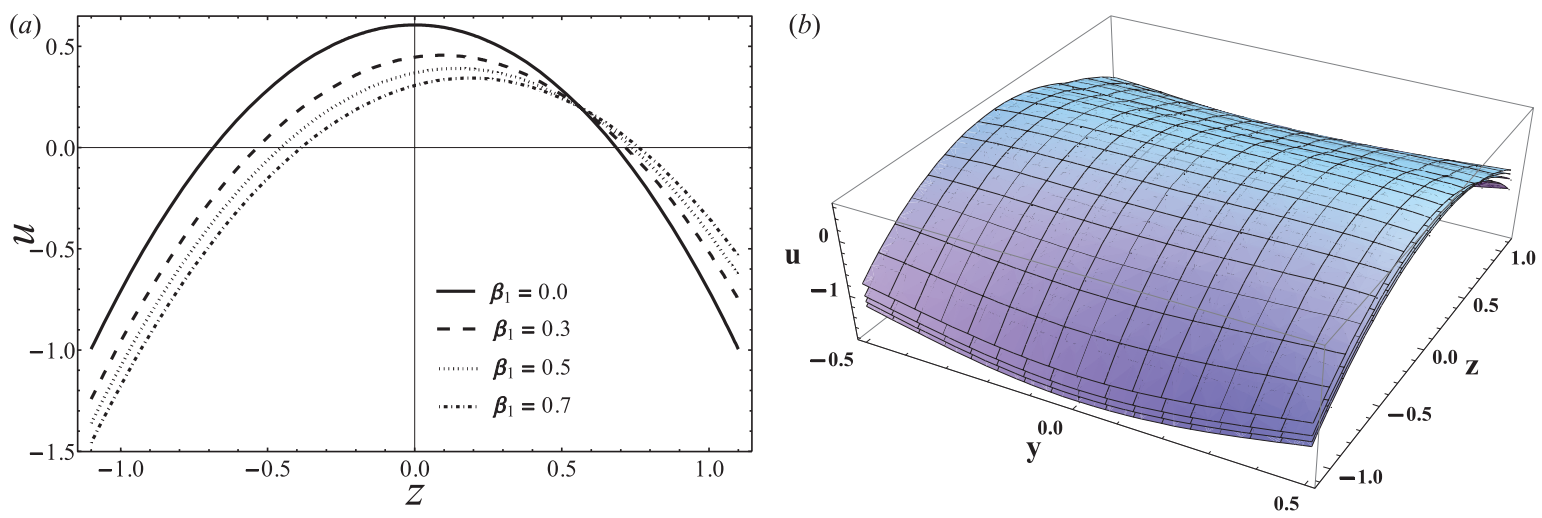

Fig. 8. Velocity profile for different values of $\beta_{1}$ for fixed $\phi=0.1, x=0, y=0.37, \beta=0.4, \lambda_{1}=2, Q=1,(a)$ for $2-$ dimensional, $(b)$ For 3-dimensional.
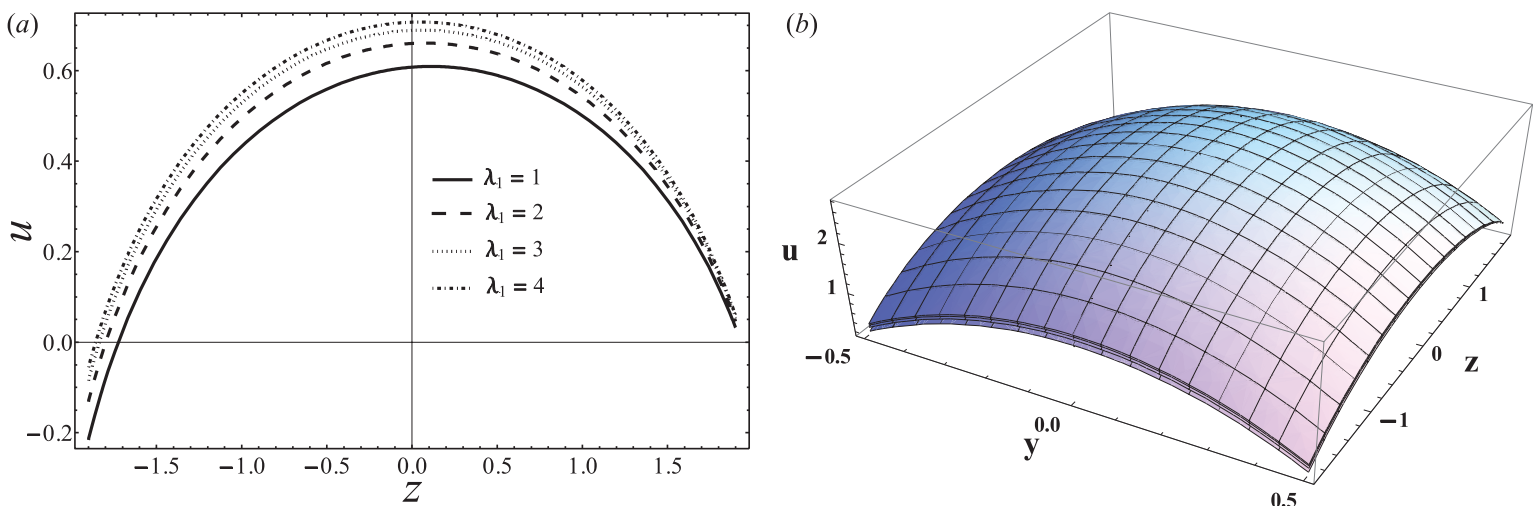

Fig. 9. Velocity profile for different values of $\lambda_{1}$ for fixed $\phi=0.9, x=0, y=0.9, \beta_{1}=0.25, \beta=0.5, Q=0.65$, (a) for $2-$ dimensional, (b) For 3-dimensional.
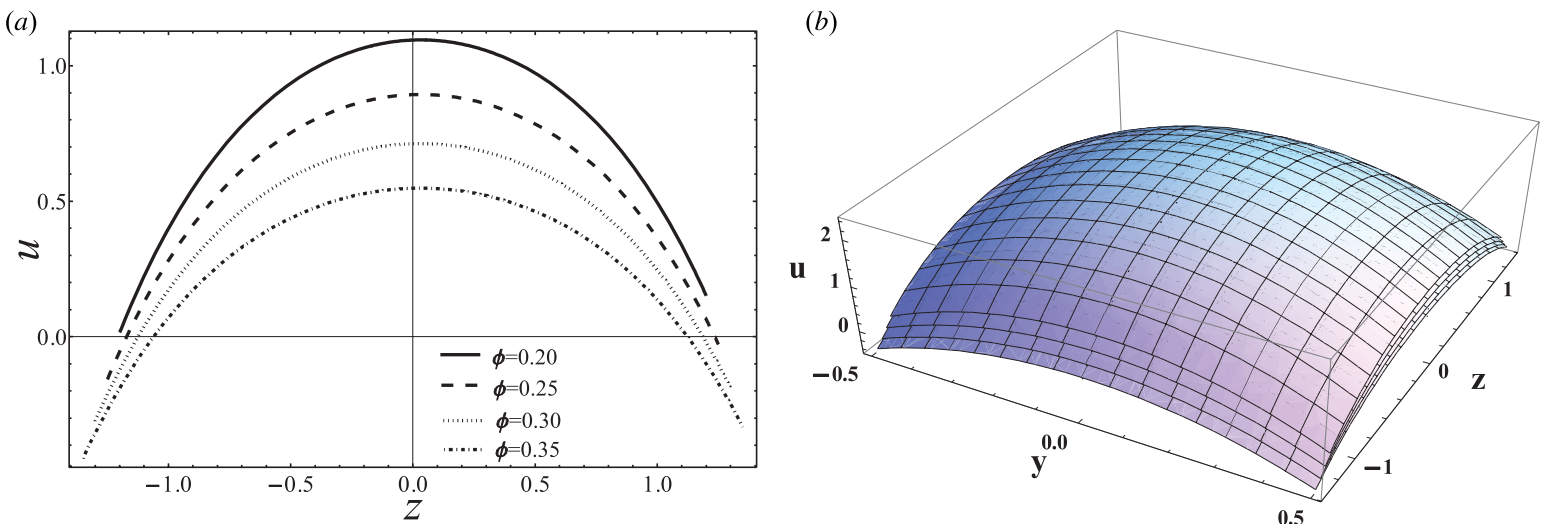

Fig. 10. Velocity profile for different values of $\phi$ for fixed $\beta=0.9, x=0, y=0.5, \beta_{1}=0.1, \lambda_{1}=2, Q=0.5,(a)$ for $2-\operatorname{dimensional,~}(b)$ For 3-dimensional. 
(a)

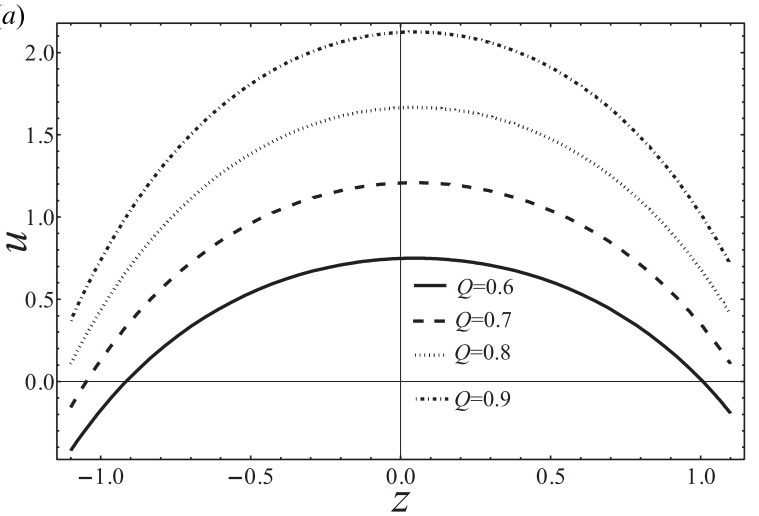

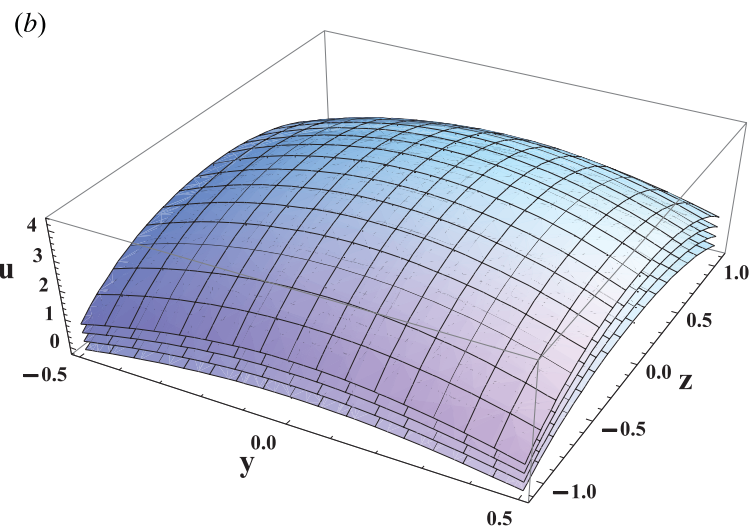

Fig. 11. Velocity profile for different values of $Q$ for fixed $\phi=0.1, x=0, y=0.5, \beta_{1}=0.15, \lambda_{1}=2, \beta=0.9$, (a) for 2-dimensional, (b) For 3-dimensional.
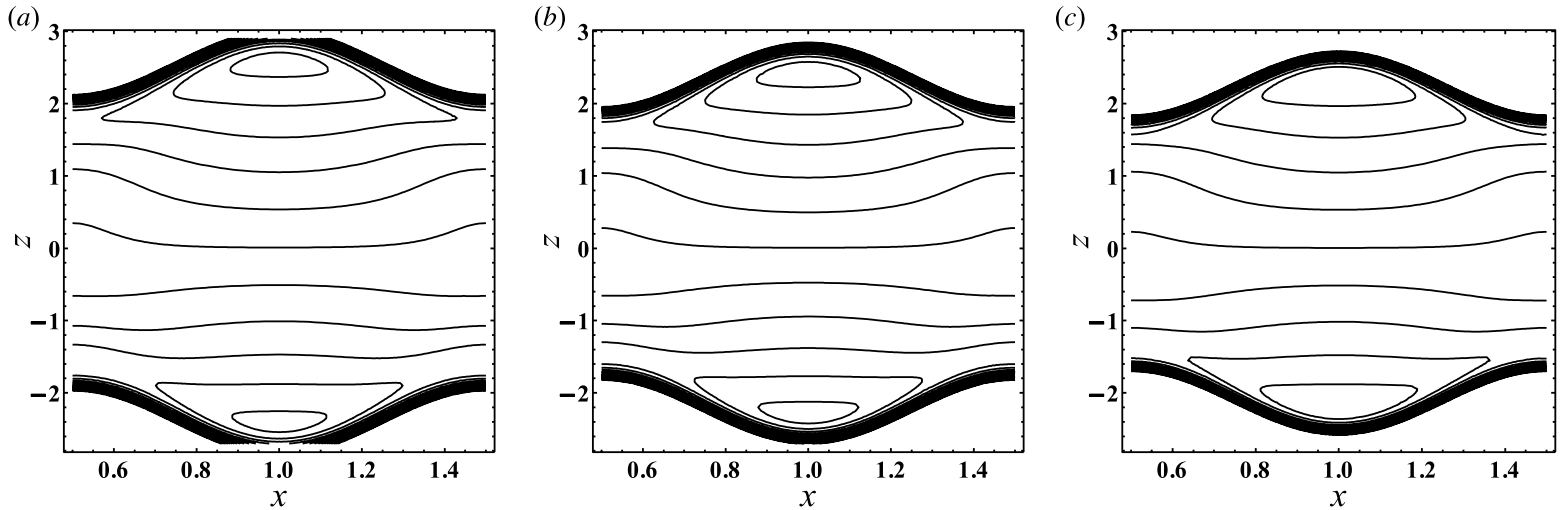

Fig. 12. Stream lines for different values of $\beta(a)$ for $\beta=0.8$, (b) for $\beta=0.9,(c)$ for $\beta=1$. The other parameters are $y=0.1, Q=0.3, \lambda_{1}=$ $0.1, \phi=0.4, \beta_{1}=0.2$

(a)

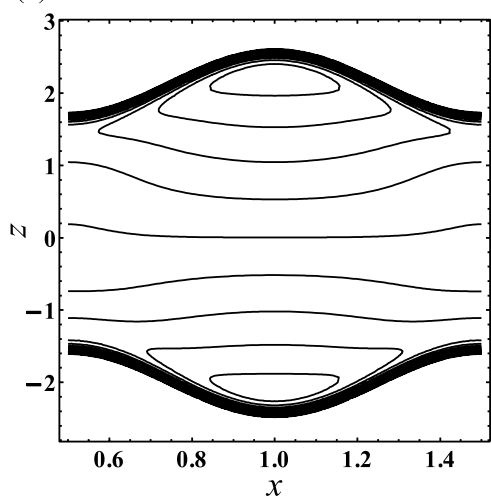

(b)

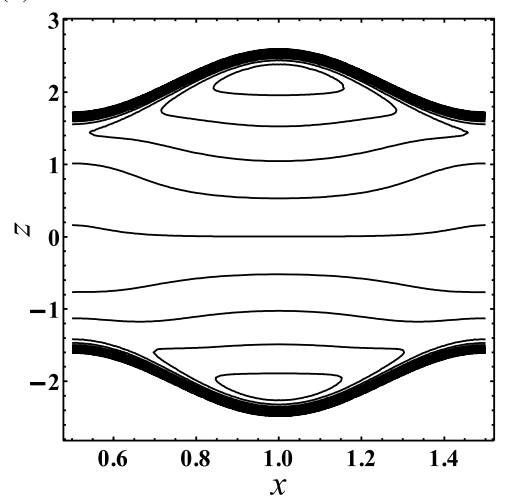

(c)

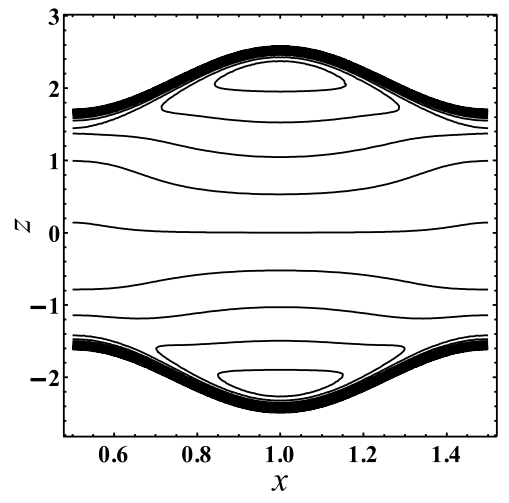

Fig. 13. Stream lines for different values of $\lambda_{1}(a)$ for $\lambda_{1}=0.1,(b)$ for $\lambda_{1}=0.5$, (c) for $\lambda_{1}=0.9$. The other parameters are $y=0.1, Q=$ $0.3, \beta=1.1, \phi=0.4, \beta_{1}=0.2$. 

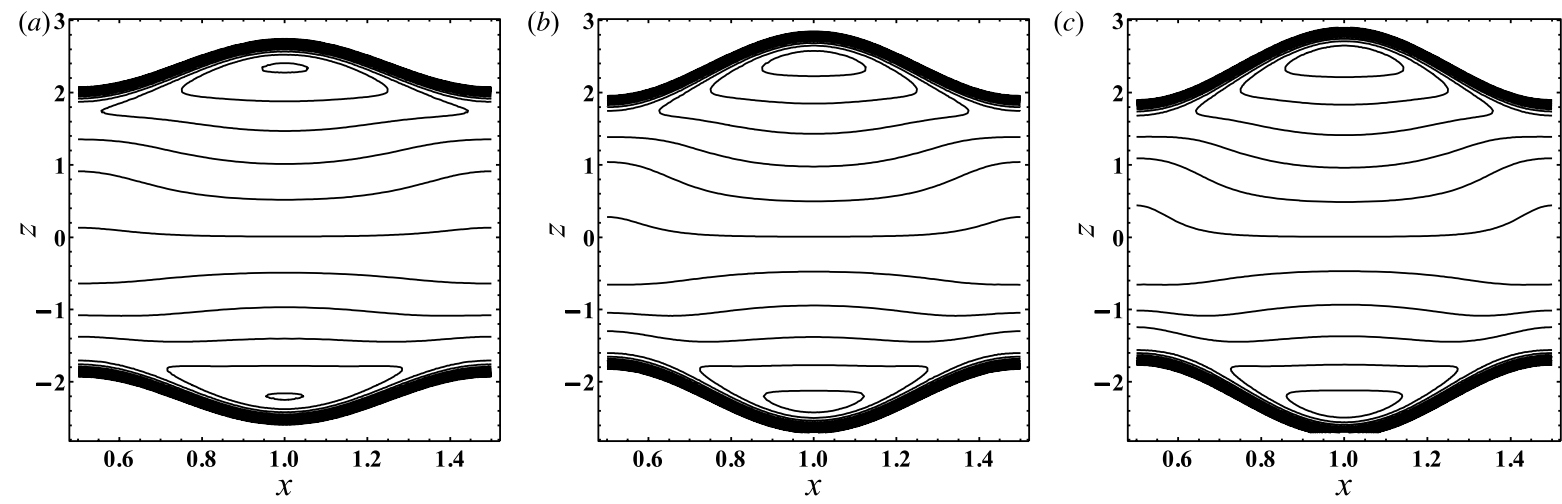

Fig. 14. Stream lines for different values of $\phi(a)$ for $\phi=0.3,(b)$ for $\phi=0.4,(c)$ for $\phi=0.45$. The other parameters are $y=0.1, Q=0.3, \beta=$ $0.9, \lambda_{1}=0.1, \beta_{1}=0.2$.
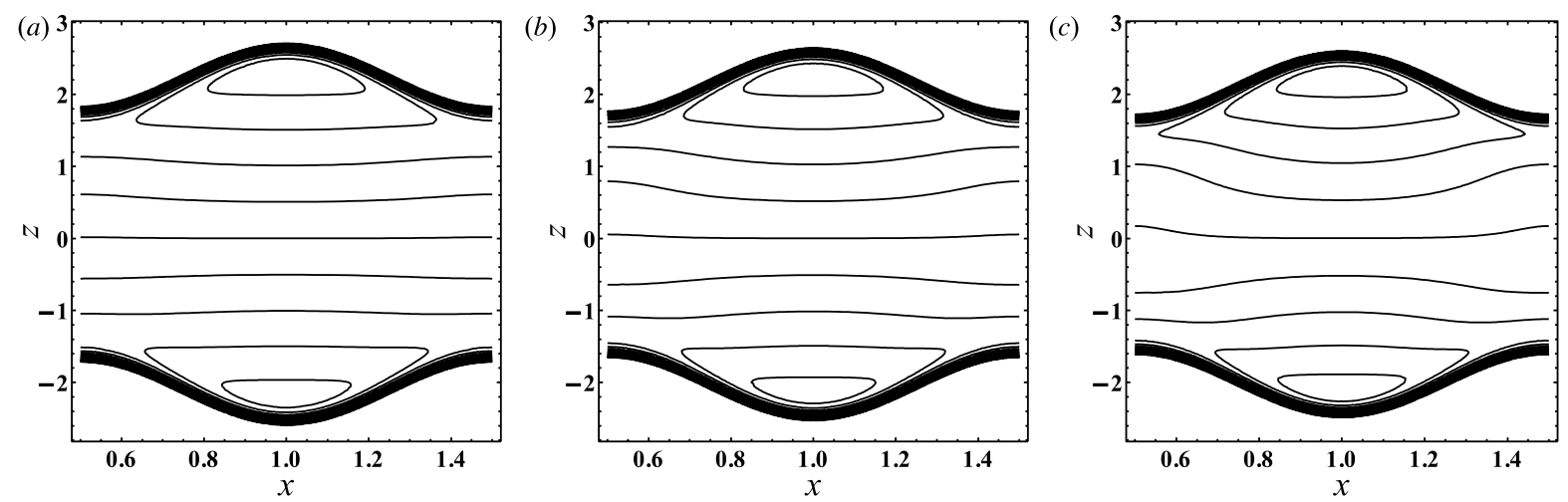

Fig. 15. Stream lines for different values of $Q(a)$ for $Q=0.1,(b)$ for $Q=0.2,(c)$ for $Q=0.3$. The other parameters are $y=0.1, \phi=$ $0.4, \beta=1.1, \lambda_{1}=0.3, \beta_{1}=0.2$.

\section{Concluding remarks}

This paper gives an account of peristaltic flow in a duct for a jeffrey fluid with partial slip effects. The equations are modeled and simplified using long wavelength and low Reynold's number approximations. The effects of various parameters on velocity $u$, pressure rise $\Delta p$, pressure gradient $d p / d x$ and trapping are discussed through graphs. We have concluded the following observations:

- The velocity field increases with the increase in $\lambda_{1}$ and $Q$ and decreases with the increase in $\beta$, $\beta_{1}$ and $\phi$.

- The rise in pressure gradient is observed with increase in $\beta_{1}$ and $\lambda_{1}$ and a reduction is observed with increase in $\beta, \phi$ and $Q$.

- Maximum resistance to the flow is observed at $x=0.5$, where as, a small pressure gradient is observed at $x<0.2$ and $x>0.8$.
- A linear dependence of pressure rise per unit wavelength is observed, pressure rise increases with increase in $\beta$ and $\phi$ and suppresses in magnitude is observed for $\beta_{1}$ and $\lambda_{1}$.

- In size of bolus formed above and below $y=0$ there is an increase with increase in $\beta$ and $\phi$ while decrease with the flow rate $Q$.

- The major role of the slip conditions is observed to slow down the flow velocity and increase the peristaltic pumping rate.

\section{References}

[1] W.M. Bayliss and E.H. Starling, The movement of innervation of small intestine, J Physiol 26 (1901), 125-138.

[2] R. Ellahi and M. Hameed, Numerical analysis of steady non-Newtonian flows with heat transfer analysis, MHD and nonlinear slip effects, Int J Numer Meth Heat Fluid Flow 22 (2012), 24-38.

[3] M. Hameed and S. Nadeem, Unsteady MHD flow of a nonNewtonian fluid on a porous plate, J Math Anal Appl $\mathbf{3 2 5}$ (2007), 724-733. 
[4] M. Hameed and R. Ellahi, Thin film flow of non-Newtonian MHD fluid on a vertically moving belt, Int J Numer Meth Fluids 66 (2011), 1409-1419.

[5] A.H. Shapiro, M.Y. Jaffrin and S.L. Weinberg, Peristaltic pumping with long wavelengths at low Reynolds number, J Fluid Mech 37 (1969), 799-825.

[6] T.W. Latham, Fluid motion in a peristaltic pump, M.Sc, Thesis, M.I.T, Cambridge, 1966.

[7] D. Tripathi, Peristaltic transport of a viscoelastic fluid in a channel, Acta Astronaut 68 (2011), 1379-1385.

[8] Y. Abdelmabouda and K.H.S. Mekheimer, Non-linear peristaltic transport of a second-order fluid through a porous medium, Appl Math Model 35 (2011), 2695-2710.

[9] D. Tripathi, A mathematical model for the peristaltic flow of chyme movement in small intestine, Math Biosci 233 (2011), 90-97.

[10] M.A. Abd Elnaby and M.H. Haroun, A new model for study the effect of wall properties on peristaltic transport of a viscous fluid, Commun Nonlinear Sci Numer Simul 13 (2008), $752-762$.

[11] K.S. Mekheimer and Y. Abdelmaboud, Peristaltic flow of a couple stress fluid in an annulus: Application of an endoscope, Physica A 387 (2008), 2403-2415.

[12] M. Kothandapani and S. Srinivas, Peristaltic transport of a Jeffrey fluid under the effect of magnetic field in an asymmetric channel, Int J Nonlinear Mech 43 (2008), 915-924.

[13] D.C. Tretheway and C.D. Meinhart, Apparent fluid slip at hydrophobic microchannel walls, Phys Fluids 14 L9 (2002), 4.

[14] C.H. Choi, K. Johan A. Westin and K.S. Breuer, Apparent slip flows in hydrophilic and hydrophobic microchannels, Phys Fluids 15 (2003), 6.

[15] E. Lauga1 and C. Cossu, A note on the stability of slip channel flows, Phys Fluids 17 (2005), 4

[16] S. Srinivas, R. Gayathri and M. Kothandapani, The influence of slip conditions, wall properties and heat transfer on MHD peristaltic transport, Comput Phys Commun 180 (2009), 2115-2122.

[17] S. Nadeem and S. Akram, Heat transfer in a peristaltic flow of MHD fluid with partial slip, Commun Nonlinear Sci Numer Simul 15 (2010), 312-321.
[18] S. Nadeem and S. Akram, Peristaltic flow of a Jeffrey fluid in a rectangular duct, Nonlinear Anal Real World Appl 11 (2010), 4238-4247.

[19] S. Nadeem, A. Riaz and R. Ellahi, Peristaltic flow of a Jeffrey fluid in a rectangular duct having compliant walls, Chem Ind Chem Engng Quart 19(3) (2013), 399-409.

[20] Kh.S. Mekheimer, S.Z. Husseny and A.I. Abdellateef, Effect of lateral walls on peristaltic flow through an asymmetric rectangular duct, Appl Bionics Biomech 8 (2011), 1-14.

[21] E. Lauga, M.P. Brenner and H.A. Stone, Microfluidics: The no-slip boundary condition, Ch. 15 in Handbook of Experimental Fluid Dynamics, Springer, New-York, 2005.

[22] M. Ansari, E. Mitsoulis and S.G. Hatzikiriakos, Capillary extrusion and swell of a HDPE melt exhibiting slip, Adv Polym Technol 32 (2013), E369-E385.

[23] L.L. Ferrás, J.M. Nóbrega, F.T. Pinho and O.S. Carneiro, Implementation of a slip boundary condition in a finite volume code aimed to predict fluid flows, II Conferência Nacional de Métodos Numéricos em Mecânica de Fluidos e Termodinâmica Universidade de Aveiro, 8-9 de Maio de 2008.

[24] N.V. Priezjev, Molecular dynamics simulations of oscillatory Couette flows with slip boundary conditions, Microfluidics and Nanofluidics 14 (2013), 225-233.

[25] J.S. Hansen and J.T. Ottesen, Molecular dynamics simulations of oscillatory flows in microfluidic channels, Microfluidics and Nanofluidics 2 (2006), 301-307.

[26] F. Sofos, T.E. Karakasidis and A. Liakopoulos, Surface wettability effects on flow in rough wall nanochannels, Microfluidics and Nanofluidics 12 (2012), 25-31.

[27] D. Kasiteropoulou, T.E. Karakasidis and A. Liakopoulos, Mesoscopic simulation of fluid flow in periodically grooved microchannel, Comput Fluids 74 (2013), 91-101.

[28] D. Kasiteropoulou, T.E. Karakasidis and A. Liakopoulos, A dissipative particle dynamics study of flow in periodically grooved nanochannels, Int J Numer Meth Fluids 68 (2012), 1156-1172.

[29] N.V. Priezjev and S.M. Troian, Influence of periodic wall roughness on the slip behaviour at liquid/solid interfaces: Molecular-scale simulations versus continuum predictions, Journal of Fluid Mechanics 554 (2006), 25-46. 

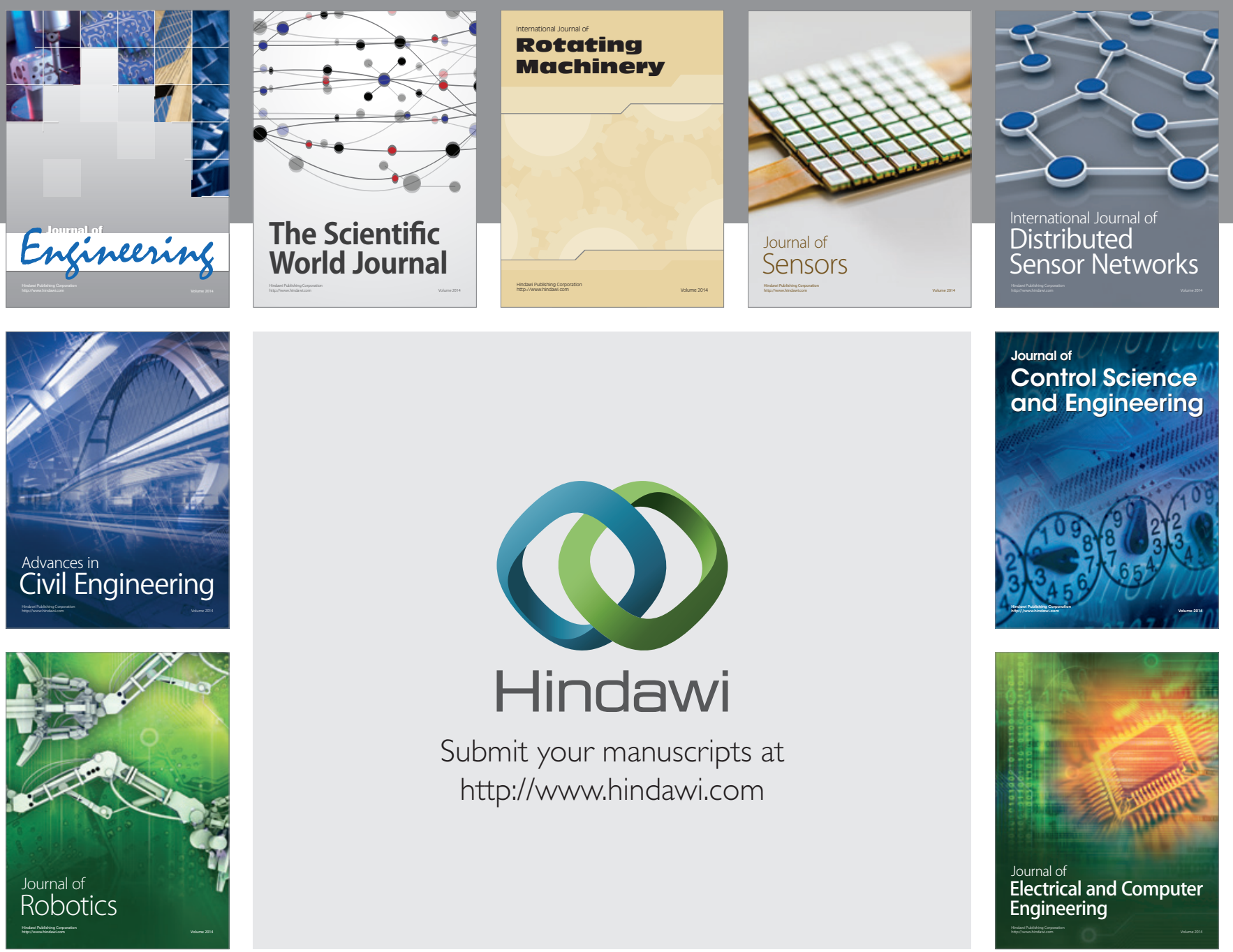

Submit your manuscripts at

http://www.hindawi.com
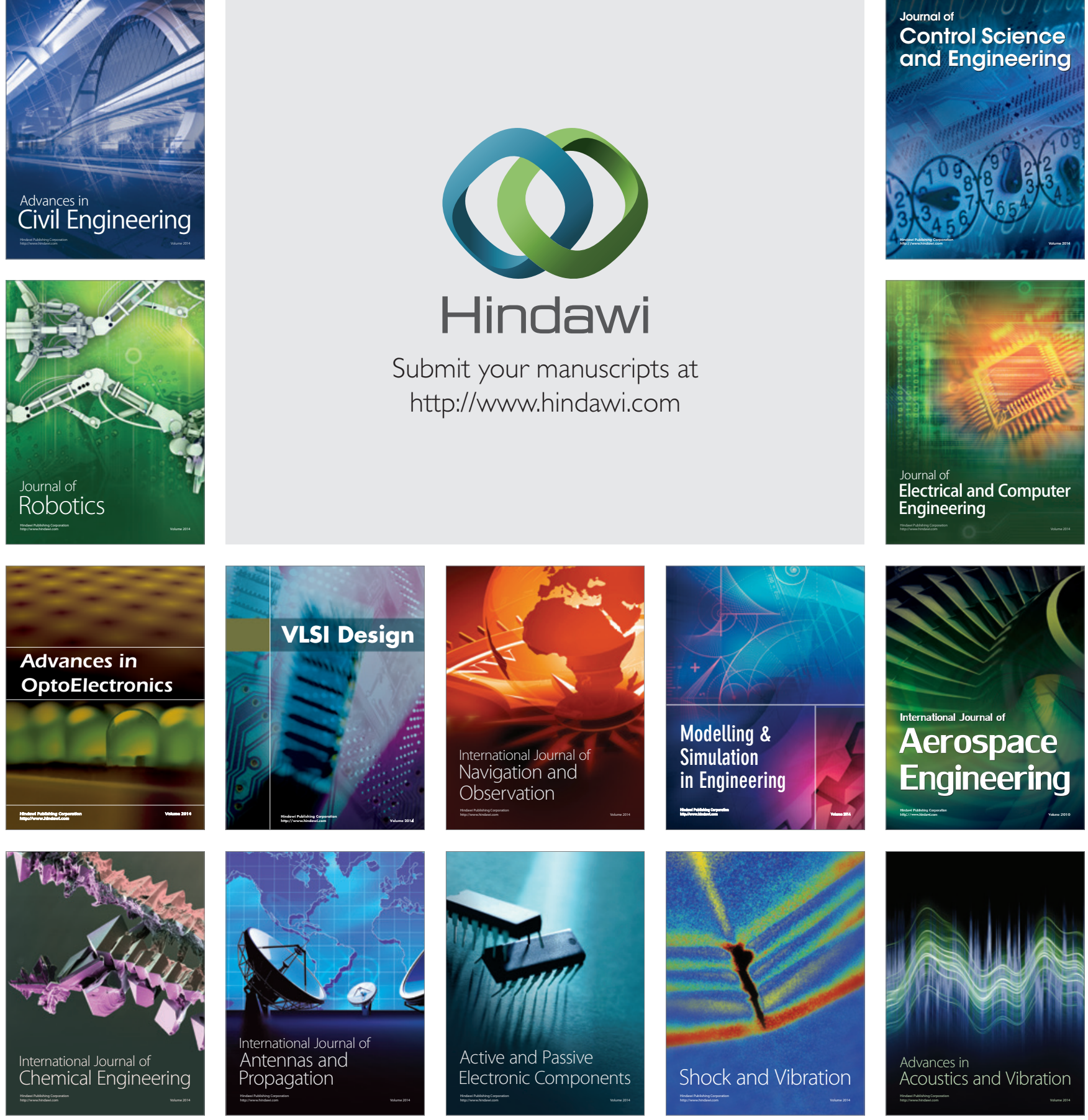